\title{
Study of Ho-doped $\mathrm{Bi}_{2} \mathrm{Te}_{3}$ topological insulator thin films
}

\author{
S. E. Harrison, ${ }^{1,2}$ L. J. Collins-McIntyre, ${ }^{1}$ S. L. Zhang, ${ }^{1}$ A. A. Baker,${ }^{1,3}$ A. I. Figueroa, ${ }^{3}$ A. J. \\ Kellock, ${ }^{4}$ A. Pushp ${ }^{4}$ Y. L. Chen,${ }^{1}$ S. S. P. Parkin, ${ }^{4}$ J. S. Harris,${ }^{2}$ G. van der Laan,${ }^{3}$ and T. Hesjedal ${ }^{1, *}$ \\ ${ }^{1}$ Department of Physics, Clarendon Laboratory, University of Oxford, Oxford, OX1 3PU, United Kingdom \\ ${ }^{2}$ Department of Electrical Engineering, Stanford University, Stanford, California 94305, USA \\ ${ }^{3}$ Magnetic Spectroscopy Group, Diamond Light Source, Didcot, OX11 ODE, United Kingdom \\ ${ }^{4}$ IBM Almaden Research Center, 650 Harry Road, San Jose, California 95120, USA
}

(Dated: September 9, 2015)

\begin{abstract}
Breaking time-reversal symmetry through magnetic doping of topological insulators (TIs) has been identified as a key strategy for unlocking exotic physical states. Doping the prototypical TIs $\mathrm{Bi}_{2} \mathrm{Te}_{3}$ and $\mathrm{Bi}_{2} \mathrm{Se}_{3}$ with rare-earth elements is particularly promising as they can offer high magnetic moments, and thus potentially large magnetic gaps in the topological surface state (TSS). Among the rare-earths, Ho has the highest magnetic moment of all elements. Here we report the molecular beam epitaxy growth of Ho-doped $\mathrm{Bi}_{2} \mathrm{Te}_{3}$ thin films. Reflection high energy electron diffraction and $\mathrm{x}$-ray diffraction studies demonstrate that high quality films were achieved for Ho doping concentrations of up to 21\%. Superconducting quantum interference device (SQUID) magnetometry shows that films are paramagnetic down to $2 \mathrm{~K}$ with a doping concentration-independent effective magnetic moment of $\sim 5 \mu_{\mathrm{B}} /$ Ho. Evidence for antiferromagnetic ordering at low temperatures was found from temperature-dependent x-ray magnetic circular dichroism and SQUID magnetometry measurements. Angle-resolved photoemission spectroscopy reveals that the TSS remained intact upon Ho doping. The large saturation moment achieved in Ho-doped TI films make them useful for incorporation into heterostructures, whereby magnetic order can be introduced via interfacial coupling.
\end{abstract}

PACS numbers: 75.30.Hx; 78.70.Dm; 75.50.Pp; 73.61.Ng

\section{INTRODUCTION}

Topological insulators (TIs) have gained strong interest as a new class of materials with fascinating yet exotic physics. ${ }^{1,2}$ They host a gapless time-reversal symmetry (TRS) protected topological surface state (TSS) that exhibits a Dirac-cone like dispersion. Breaking the TRS in TIs through magnetic doping can result in gap forming at the Dirac-point in the TSS. ${ }^{3,4}$ This altered bandstructure may host a variety of interesting physical phenomena, such as the quantum anomalous Hall effect (QAHE). ${ }^{5}$ The prediction of novel quantized states arising from the peculiar coupling between magnetic and electric fields ${ }^{6}$ make ferromagnetic TIs an intriguing venue for the next generation electronic and spintronic devices. Potential applications of ferromagnetic TIs include interconnects, memory cells, and other novel electronic devices. ${ }^{7-10}$

The three-dimensional TIs $\mathrm{Bi}_{2} \mathrm{Te}_{3}$ and $\mathrm{Bi}_{2} \mathrm{Se}_{3}$ are the most thoroughly investigated class of TI materials due to their (comparably) large bulk bandgap of 0.15-0.3 eV and simple single Dirac-cone surface states. ${ }^{11}$ Ferromagnetic order has been achieved by transition metal doping, ${ }^{12}$ e.g., with $\mathrm{Fe},{ }^{13} \mathrm{Mn},{ }^{14-18}$ and $\mathrm{Cr},{ }^{19-21}$ of bulk and thin film samples. A major concern for magnetic TI doping, however, is to avoid the introduction of additional charge carriers in the material. In principle, transition metal dopants such as Cr should enter the host in their $3+$ state, substituting for $\mathrm{Bi}$, however, recent $\mathrm{x}$-ray spectroscopy found $\mathrm{Cr}$ to be divalent in $\mathrm{Bi}_{2} \mathrm{Se}_{3} \cdot{ }^{22,23}$ Doping with most rare-earth ( $\mathrm{RE}$ ) ions, on the other hand, has the advantage of substituting $\mathrm{Bi}^{3+}$ iso-electronically with $\mathrm{RE}^{3+}$, as well as introducing a large magnetic moment into the system which may lead to increased magnetic effects. The effective magnetic moment of $\mathrm{Gd}^{3+}$, which has the maximum number of 7 unpaired $f$ electrons in the lanthanide series, is $7.94 \mu_{\mathrm{B}}$. However, even higher effective moments of $10.6 \mu_{\mathrm{B}}$ and $10.4 \mu_{\mathrm{B}}$ are found for $\mathrm{Dy}^{3+}\left(4 f^{9}\right)$ and $\mathrm{Ho}^{3+}\left(4 f^{10}\right)$, respectively, due to spinorbit coupling.

Recently, we presented the growth of high quality rhombohedral Gd- and Dy-doped $\mathrm{Bi}_{2} \mathrm{Te}_{3}$ thin films by molecular beam epitaxy (MBE) with high RE concentrations (up to $40 \%$ of the Bi sites), exceeding reported bulk solubility limits. ${ }^{24-27}$ Systematic material properties studies on both doping systems revealed a variety of similarities as well as remarkable differences. For both Gd- and Dy-doped films, long-range magnetic order was not observed in magnetometry as films remained paramagnetic down to $2 \mathrm{~K}$. However, whereas the magnetic moment in Gd-doped films is doping concentration independent, an strong decrease was observed as a function of Dy concentration. In addition, for all Gd-doping concentrations investigated angle-resolved photoemission spectroscopy (ARPES) confirmed the existence of an intact TSS, whereas above a critical limit for Dy-doping, evidence for a gapped TSS was observed. Intriguing results, such as these, from previous work motivated additional RE-doping experiments using the highest magnetic moment element of all elements, Ho. In this letter, we present the MBE growth and structural, magnetic, and electronic properties study of $\left(\mathrm{Ho}_{x} \mathrm{Bi}_{1-x}\right)_{2} \mathrm{Te}_{3}$ topological insulator thin films which aims to expand on current understanding of the effects of RE-doping in TIs. 


\section{THIN FILM GROWTH}

$\left(\mathrm{Ho}_{x} \mathrm{Bi}_{1-x}\right)_{2} \mathrm{Te}_{3}$ thin films $(0 \leq x \leq 0.21)$ were grown on $c$-plane sapphire substrates using MBE. As-received, 2"diameter $\mathrm{Al}_{2} \mathrm{O}_{3}$ (0001) wafers were cleaved into quarters, degreased in hot solvents and outgassed by baking under ultra-high vacuum for $8 \mathrm{~h}$ at $150^{\circ} \mathrm{C}$. Prior to growth, cleaned substrates were introduced into the main growth chamber with a base pressure of $\sim 5 \times 10^{-11}$ Torr. The substrate temperature, determined by a thermocouple, was ramped up to $450^{\circ} \mathrm{C}$ and held at temperature for $\sim 10 \mathrm{~min}$ before ramping down to the starting growth temperature. Thin film deposition was carried out by co-evaporation of high purity Bi (99.9999\%), Te (99.9999\%), and Ho (99.99\%) from standard Knudsen effusion cells. The Ho concentration, $x$, was controlled by varying the Ho effusion cell temperature $\left(T_{\mathrm{Ho}}=840\right.$ $900^{\circ} \mathrm{C}$ ), while the $\mathrm{Bi}$ and $\mathrm{Te}$ cell temperatures were held constant $\left(458^{\circ} \mathrm{C}\right.$ and $235^{\circ} \mathrm{C}$, respectively), which resulted in a nominal Te:Bi ratio of $\sim 15$. The $\mathrm{Ho}, \mathrm{Bi}$, and Te cell fluxes were calibrated using an ion gauge beam flux monitor.

A two-temperature-step process, ${ }^{28}$ similar to the recipe described in Refs. 26, 27, and 29, was used for growth of the $\left(\mathrm{Ho}_{x} \mathrm{Bi}_{1-x}\right)_{2} \mathrm{Te}_{3}$ thin films. During the first growth step, a low temperature nucleation layer was deposited for 33 min at $250^{\circ} \mathrm{C}$. Film growth was then paused while the substrate temperature was ramped up to $300^{\circ} \mathrm{C}$ under Te flux at a rate of $5^{\circ} \mathrm{C} \mathrm{min}{ }^{-1}$. At $300^{\circ} \mathrm{C}$, the sample was annealed for 30 min before growth continued at $300^{\circ} \mathrm{C}$ for another $33 \mathrm{~min}$. After growth was terminated, samples were allowed to cool to room temperature in ultra-high vacuum before being unloaded for ex situ characterization.

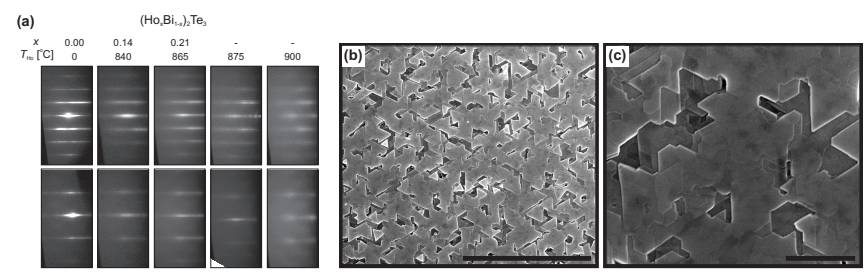

FIG. 1. (a) RHEED patterns of $\left(\mathrm{Ho}_{x} \mathrm{Bi}_{1-x}\right)_{2} \mathrm{Te}_{3}$ films on $c$-plane sapphire for the Ho cell temperatures and concentrations as indicated. Patterns obtained along the [1010] azimuth (lower row) and along the $[11 \overline{2} 0]$ azimuth (upper row) of $\mathrm{Al}_{2} \mathrm{O}_{3}(0001)$. (b,c) SEM images for an $x=0.14$ film. The scale bar in (b) represents $5 \mu \mathrm{m}$ and in (c) $500 \mathrm{~nm}$.

In situ reflection high energy electron diffraction (RHEED) was used to provide real-time feedback about the surface morphology of the growing epilayer as a function of the Ho cell temperature. Films were found to exhibit two distinct RHEED patterns along the [1010] and $[11 \overline{2} 0]$ azimuth of the $\mathrm{Al}_{2} \mathrm{O}_{3}$ (0001) substrate. These patterns were found to repeat upon $60^{\circ}$ rotation. As shown in Fig. 1(a), for Ho cell temperatures up to $865^{\circ} \mathrm{C}$, the RHEED patterns observed were well-defined with a streak-like appearance characteristic of a planar surface morphology. At Ho cell temperatures above $865^{\circ} \mathrm{C}$, however, the RHEED patterns showed evidence for degradation as the RHEED streaks became more diffuse and developed a spot-like appearance which are indicative of increasing surface disorder and the onset of 3D growth. As a result of these findings, we choose to focus the rest of this study on thin films grown with Ho cell temperatures up to $865^{\circ} \mathrm{C}$. In addition to RHEED, the surface morphology of the Ho-doped films was also further investigated by ex situ measurements using scanning electron microscopy (SEM). Typical examples of large-area and small-area scans, showing the characteristic triangular domain structure known from undoped and doped $\mathrm{Bi}_{2} \mathrm{Te}_{3}$ thin films, ${ }^{26}$ are shown in Figs. 1(b) and 1(c), respectively, for a film grown with $T_{\mathrm{Ho}}=840^{\circ} \mathrm{C}$.

TABLE I. Composition and thickness $t$ determined by RBS and PIXE measurements for thin films grown at the indicated Ho cell temperatures, $T_{\text {Ho }}$. The error for the elemental compositions is 0.5 at- $\%$ for Se and all elements for the nominally undoped film, and 2 at-\% otherwise.

\begin{tabular}{ccccccc}
\hline $\begin{array}{c}T_{\mathrm{Ho}} \\
\left({ }^{\circ} \mathrm{C}\right)\end{array}$ & $x_{\mathrm{Ho}}$ & $\begin{array}{c}\mathrm{Ho} \\
(\text { at-\%) }\end{array}$ & $\begin{array}{c}\mathrm{Bi} \\
(\text { at-\% })\end{array}$ & $\begin{array}{c}\mathrm{Te} \\
(\text { at-\% })\end{array}$ & $\begin{array}{c}\mathrm{Se} \\
(\text { at-\% })\end{array}$ & $\begin{array}{c}t \\
(\AA)\end{array}$ \\
\hline- & 0 & 0.0 & 39.2 & 59.7 & 1.1 & $721 \pm 50$ \\
840 & 0.14 & 5.5 & 35.8 & 56.4 & 2.3 & $800 \pm 100$ \\
865 & 0.21 & 8.4 & 33.4 & 55.3 & 2.9 & $941 \pm 100$ \\
\hline \hline
\end{tabular}

Compositional analysis was carried out using a combination of Rutherford backscattering spectroscopy (RBS) with $2.3 \mathrm{MeV}$ He ions and particle induced x-ray emission (PIXE) with $1 \mathrm{MeV} \mathrm{H}$ ions. The combined approach was performed due to smaller backscattered ion energy differences in the measured RBS spectra for heavy elements. For materials that composed of more than one heavy element, such as RE-doped $\mathrm{Bi}_{2} \mathrm{Te}_{3}$, this can lead to peak overlap and uncertainty in the composition determination. For our studies, the peak overlap between the constituent heavy elements was resolved using PIXE. In PIXE, heavy elements have distinct energy differences in the spectra which are not present in RBS.

The combined RBS/PIXE results are summarized in Tab. I. Note that a small Se concentration was detected as well, which resulted from a Se background contamination of the MBE growth chamber. In all cases, the Se and Te concentrations were found to add up to $\sim 60$ at$\%$ (atomic-\%; within error), which suggests that Se is replacing $\mathrm{Te}$ in the lattice. In addition, analysis of the cation $(\mathrm{Ho}+\mathrm{Bi})$ to anion $(\mathrm{Te}+\mathrm{Se})$ ratios shows a relationship of $\sim 2: 3$, within the specified error margin, which is indicative of Ho being mostly substitutional on $\mathrm{Bi}$ sites for the doping series. ${ }^{24}$ 


\section{STRUCTURAL PROPERTIES}

X-ray diffraction (XRD) measurements were performed to investigate the effects of Ho incorporation on the structural properties of the $\mathrm{Bi}_{2} \mathrm{Te}_{3}$ host. Figure 2(a) shows symmetric $2 \theta-\omega$ scans obtained with a PANalytical X'Pert PRO diffractometer using $\mathrm{Cu} \mathrm{K} \alpha_{1}$ emission and a triple-axis detector in grazing-incidence configuration. Only substrate and film peaks corresponding to the relative positions of the $\mathrm{Bi}_{2} \mathrm{Te}_{3}$ (00l) family of diffraction peaks were observed which indicates that the $\left(\mathrm{Ho}_{x} \mathrm{Bi}_{1-x}\right)_{2} \mathrm{Te}_{3}$ thin films are $c$-axis oriented and grow in a rhombohedral crystalline structure. In addition, the absence of additional peaks in the spectra indicates that no secondary phases were detected up to $x=0.21$.
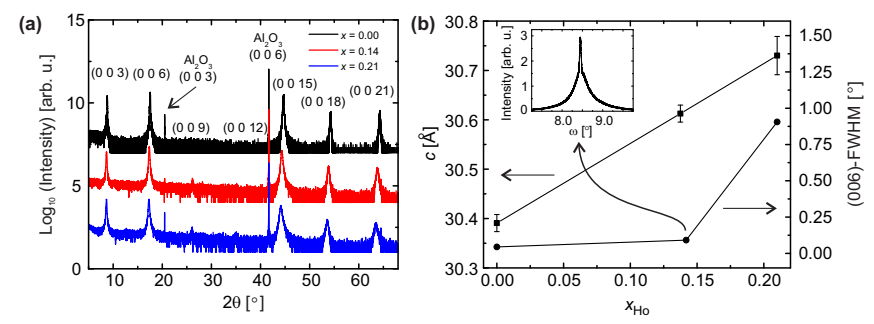

FIG. 2. XRD measurements. (a) $2 \theta-\omega$ scans obtained on an undoped binary $\mathrm{Bi}_{2} \mathrm{Te}_{3}$ thin film (black), and $\left(\mathrm{Dy}_{x} \mathrm{Bi}_{1-x}\right)_{2} \mathrm{Te}_{3}$ films with $x=0.14$ (red) and 0.21 (blue). The $\left(\begin{array}{lll}0 & l\end{array}\right)$ film and substrate peaks are labeled. (b) Left axis: Out-of-plane lattice parameter $c$ obtained from the $\left(\begin{array}{lll}0 & 0 & 3 l\end{array}\right)$ reflections. Right axis: FWHM of the (006) rocking curves. Inset: example of a (006) rocking curve for $x=0.14$.

Careful analysis of the Bragg peaks in Fig. 2(a) reveals that the peaks shift toward lower diffraction angles and experience broadening as a function of doping concentration, which are consistent with observations for other MBE-grown RE-doped $\mathrm{Bi}_{2} \mathrm{Te}_{3}$ thin films. ${ }^{24,26,27}$ The appearance of these effects is indicative of an increased outof-plane lattice constant and degradation in overall crystalline quality with increasing Ho doping. The expansion of the out-of-plane lattice parameter $c$, determined from the $2 \theta$ values of the $(00 l)$ peaks for $5^{\circ}-65^{\circ}$ using a nonlinear least-square cell-refinement program, is plotted in Fig. 2(b) (left axis). The increase of $\sim 0.3 \AA$ at $x=0.21$ is slightly larger than the increase in $c$ for Dy-doped films with similar $x .^{27}$

Evidence of the degradation of crystallinity is further observed in the increase in the full-width at half maximum (FWHM) of the (006) rocking curves, as shown in Fig. 2(b) (right axis). Films with $x=0.14$, however, were found to be of relatively high crystalline quality with only an increase of $\sim 0.046^{\circ}$ in the (006) FWHM when compared to the undoped film. In addition, when compared to other magnetically-doped TIs films with similar doping concentrations, the FWHM of the (006) rocking curve for the $x=0.14$ sample was $\sim 7.50 \times$ smaller and $\sim 0.57 \times$ larger than the values obtained for Cr-doped ${ }^{21}$ and Dy-doped films, respectively. Additional XRD measurements, provided in the Supplementary Material, ${ }^{30}$ were also performed which expand upon and further support these findings (see the 2D asymmetric reciprocal space mappings (RSM) in Supplementary Fig. 1).

\section{MAGNETIC PROPERTIES}

\section{A. SQUID magnetization measurements}

Bulk magnetization measurements were carried out on the Ho-doped samples using a superconducting quantum interference device (SQUID) magnetometer (7 T Quantum Design MPMS SQUID VSM). Figure 3(a) shows $M(H)$ plots obtained at $2 \mathrm{~K}$ for films with $x=0.14$ and $x=0.21$ in an applied magnetic field ranging between \pm 7 T. Measurements were performed with the field applied perpendicular to the $c$-axis, i.e., in-plane. In order to isolate the film signals, the diamagnetic background from the sapphire substrate was removed via high-field linear fitting. The shape of the magnetization curves resembles the Brillouin function which is indicative of paramagnetic behavior. The magnetic moments per Ho ion [Fig. 3(a)] were obtained using the film volume, determined from the mass of the sample and the RBS/PIXE thickness measurements, and the Ho concentration from the RBS/PIXE data. The saturation moments were found to be $(5.20 \pm 0.74) \mu_{\mathrm{B}} /$ Ho and $(5.08 \pm 0.60) \mu_{\mathrm{B}} / \mathrm{Ho}$ for $x=0.14$ and $x=0.21$, respectively. The stated errors are determined by the uncertainty in the determination of sample volume from the RBS/PIXE data. The moments were found to be fully saturated at $\sim 4 \mathrm{~T}$ (at $2 \mathrm{~K}$ ), and, within the errors, independent of the doping concentration. The observed moments are significantly less than the theoretical free-ion value of $\sim 10.6 \mu_{\mathrm{B}}$ for $\mathrm{Ho}^{3+}$ and may arise from oxidation of the Ho dopants, crystalfield effects and/or the appearance of antiferromagnetic coupling. ${ }^{20,31,32}$

In terms of the doping concentration dependence of the magnetic moment, Ho-doped films behave like Gddoped $\mathrm{Bi}_{2} \mathrm{Te}_{3}$, however, in case of $\mathrm{Gd}$ doping the full spin moment was observed. ${ }^{25}$ In contrast, studies on Dydoped $\mathrm{Bi}_{2} \mathrm{Te}_{3}$ films revealed a concentration-dependent saturation magnetization, which was found to increase with decreasing doping concentration, as well as sample quality. ${ }^{26}$

Magnetization vs temperature measurements in an applied field of $100 \mathrm{mT}$, shown in Fig. 3(b) for a film with $x=0.14$, were also performed. All films in the doping series yielded $M(T)$ plots that exhibited a typical paramagnetic response. A plot of the inverse susceptibility, $1 / \chi$, is shown in the inset in Fig. 3(b). In order to highlight deviations from the paramagnetic behavior, the susceptibility was fitted using the Curie-Weiss dependence, $\chi=C /(T-\Theta)$ where $C$ is the material-specific Curie constant and $\Theta$ the Weiss temperature. The fit for $x=0.14$ 
is shown by a red line in Fig. 3(b) and reveals a negative Weiss temperature $(\Theta=-0.9 \mathrm{~K})$ which points towards possible antiferromagnetic ordering at low temperatures. Similar results were also obtained on Ho-doped films with $x=0.21$.
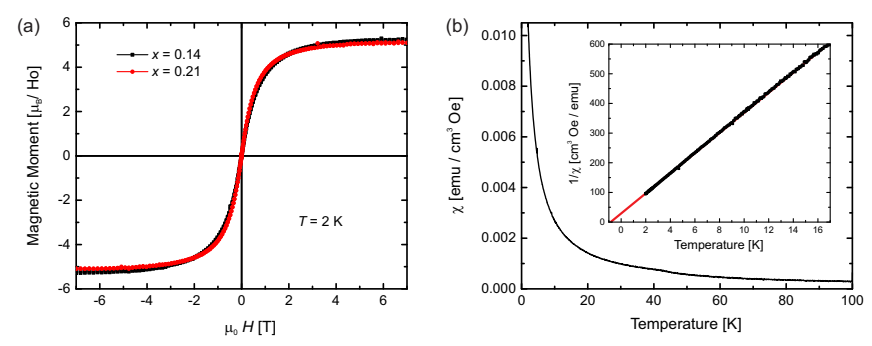

FIG. 3. SQUID magnetometry. (a) $M(H)$ plots obtained at $2 \mathrm{~K}$ for $x=0.14$ and $x=0.21$ with the field applied in-plane. (b) Plot of the susceptibility, $\chi$, as a function of temperature. Inset: Plot of $1 / \chi$ for the $x=0.14$ sample. The intercept $\Theta$ is negative $(\approx-0.9 \mathrm{~K})$.

\section{B. X-ray magnetic circular dichroism}

The element-specific technique of XMCD is used to probe the local electronic character of the magnetic ground state. ${ }^{33}$ This technique allows an unambiguous determination of the electronic and magnetic state of transition metal and rare-earths magnetic dopants in TIs. ${ }^{17,25,26,34}$

X-ray absorption spectra (XAS) at the Ho $M_{4,5}$ edges were measured on beamline I10 (BLADE) at the Diamond Light Source, Oxfordshire, using a 14 T superconducting magnet. XAS measurements were made in totalelectron-yield (TEY) mode. The XMCD is obtained from the difference between two XAS spectra recorded with the x-ray helicity vector and applied magnetic field parallel and antiparallel, respectively. ${ }^{35}$ The magnetic field is always parallel to the x-ray beam and the samples (with the normal || $c$-axis) were measured at both normal and grazing incidence. The XMCD is measured by reversing the polarization of the incident $\mathrm{x}$-rays to avoid having to change the magnetic field of the superconducting magnet.

Unlike the $5 d$ and $6 s$ electrons in Ho, the $4 f$ electrons are not directly involved in the chemical bonding. Therefore, the Ho $M_{4,5}$ spectrum is essentially the same for the metal and alloys, as well as oxides and compounds, apart from small differences in line broadening. Since the additional $4 f$ electron is effectively screening the $3 d$ hole, the chemical shift in the $M_{4,5}$ spectra is small, so that no chemical information is obtained from the XAS and XMCD spectra. However, the magnitude of the $\mathrm{XMCD}$ signal is directly proportional to the $4 f$ magnetic moment. ${ }^{36,37}$

Figure $4(\mathrm{a})$ shows the Ho $M_{5}$ XMCD for the Hodoped $\mathrm{Bi}_{2} \mathrm{Te}_{3}$ thin film with $x=0.14$, measured over a
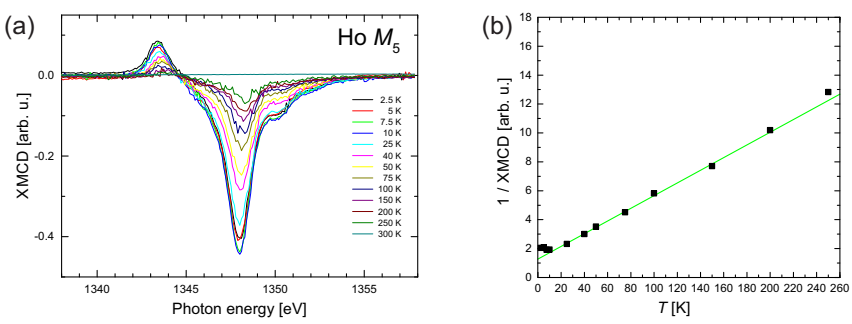

FIG. 4. Temperature-dependent XMCD measurements of Hodoped $\mathrm{Bi}_{2} \mathrm{Te}_{3}$ thin film with $x=0.14$. (a) Ho $M_{5} \mathrm{XMCD}$ measured in TEY at normal incidence in an applied field of $7 \mathrm{~T}$ for a range of temperatures. (b) Inverse susceptibility derived from the XMCD signal. The green straight line through the TEY XMCD suggests a negative Weiss temperature.

range of temperatures with an out-of-plane applied field of $7 \mathrm{~T}$. The moment is zero at $300 \mathrm{~K}$, and gradually increases for decreasing temperature. It reaches a maximum around around $7 \mathrm{~K}$, after which it reduces again going further down in temperature. An estimation of the Weiss temperature may be obtained by plotting the inverse of the magnetic susceptibility $1 / \chi=H / M$ (using the moment obtained from XMCD) as a function of temperature [Fig. 4(b)]. This shows the typical linear dependency expected for a paramagnetic system with a small deviation at lower temperatures. Linear extrapolation leads to a negative Weiss temperature, which means the material is always paramagnetic above $0 \mathrm{~K}$.

\section{ELECTRONIC PROPERTIES}

Angle-resolved photoemission spectroscopy (ARPES) measurements were performed to investigate the electronic structure of the Ho-doped films. Figure 5 shows bandstructure data obtained at $20 \mathrm{~K}$ on an in situ cleaved Ho-doped $\mathrm{Bi}_{2} \mathrm{Te}_{3}$ thin film with $x=0.14 .^{38}$ The presence of the topological surface state band (SSB) is resolved in $3 \mathrm{D}$ representation shown in Fig. 5(a). The Fermi velocity obtained along the $\Gamma-\mathrm{K}$ direction was found to be approximately $4.7 \times 10^{5} \mathrm{~m} \mathrm{~s}^{-1}(2.5 \mathrm{eV} \AA)$ which is similar to values reported for undoped single-crystal and thin film $\mathrm{Bi}_{2} \mathrm{Te}_{3}$ [Refs. 28 and 39] as well as Gd-doped $\mathrm{Bi}_{2} \mathrm{Te}_{3}$ thin films. ${ }^{24,38}$ Stacked constant energy mappings showing the evolution of the SSB and bulk valance band (BVB) for different binding energies are shown in Fig. 5(b). At more negative binding energies, the disappearance of the SSB and the emergence of the hexagram-like BVB are observed and consistence with previous reports on $\mathrm{Bi}_{2} \mathrm{Te}_{3} \cdot{ }^{39}$ Note that the bulk conduction band (BCB) is not visible in data shown in Fig. 5 due to the relative position of the Fermi level.

Bandstructure data obtained at XXXX eV along the $\Gamma-\mathrm{K}$ direction is shown in Fig. 5(c), where the surface state band is indicated in red. The energy distribution curve (EDC) at $k_{\|}=0$ is provided in Fig. $5(\mathrm{~d})$. No in- 

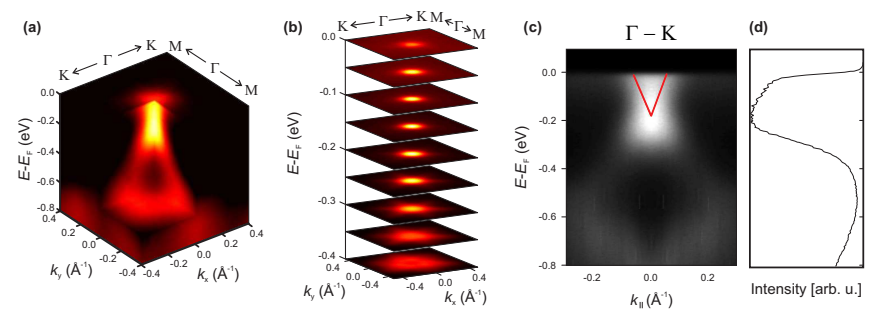

FIG. 5. Band structures of an in situ cleaved Ho-doped thin film with $x=0.14$. (a) $3 \mathrm{D}$ bandstructure plot showing the 2D Brillouin zone. (b) Stack of constant energy maps at the binding energies, $E-E_{F}$. Both the surface state band (SSB) and the bulk valence band (BVB) are resolved. The BVB shows a six-fold symmetry. (c) Cut of the 3D bandstructure along the $\Gamma-\mathrm{K}$ direction, and (d) intensity profile (energy distribution curve) at $k_{\|}=0$. No intensity suppression is seen in the bandstructure near the Dirac point.

dication of a magnetic doping-induced gap at the Dirac point was observed, which should be visible as a intensity suppression in Fig. 5(c,d), meaning that TRS is not broken. In this sense, the Ho-doped films are comparable to the Gd-doped films, ${ }^{28}$ but different than the Dy-doped films in which a gap has been observed, ${ }^{27}$ despite the absence of magnetic long range order. ${ }^{26}$ Additional ARPES data are provided in the Supplementary Fig. $2 .{ }^{30}$

\section{SUMMARY AND CONCLUSIONS}

In conclusion, we presented a systematic study of the structural, magnetic, and electronic properties of high-quality MBE-grown $\left(\mathrm{Ho}_{x} \mathrm{Bi}_{1-x}\right)_{2} \mathrm{Te}_{3}$ thin films. Ho was found to incorporate substitutionally on Bi sites of the rhombohedral crystal without the formation of secondary phases for Ho concentrations up to 21 at- $\%$. The films were found to be paramagnetic down to the lowest probed temperature $(2 \mathrm{~K})$ with a doping concentrationindependent effective magnetic moment of $\sim 5 \mu_{\mathrm{B}}$, determined by magnetometry. Evidence for possible antiferromagnetic ordering with a negative Weiss temperature was deduced from SQUID and XMCD measurements. Electronic bandstructure measurements revealed that the topological surface state remained intact upon Ho doping, further indicating the absence of TRS-breaking in the Ho-doped system. Despite the absence of TRSbreaking magnetic order, Ho-doped films may still prove useful, when proximity-coupled to a ferromagnet, for achieving the QAHE state.

\section{ACKNOWLEDGMENTS}

This publication arises from research funded by the John Fell Oxford University Press (OUP) Research Fund and the Research Complex at Harwell is acknowledged for their hospitality. This work was supported by a DARPA MESO project (No. N66001-11-1-4105). SEH was supported by the VPGE (Stanford University). LCM and AAB acknowledge partial financial support from EPSRC (UK) through a Doctoral Training Award. Diamond Light Source is acknowledged for beamtime on I10 (proposal SI10207).
* Corresponding author: Thorsten.Hesjedal@physics.ox.ac.uk

1 C. Kane and E. Mele, Phys. Rev. Lett. 95, 146802 (2005).

2 B. A. Bernevig, T. L. Hughes, and S.-C. Zhang, Science 314, 1757 (2006).

3 Y. L. Chen, J.-H. Chu, J. G. Analytis, Z. K. Liu, K. Igarashi, H.-H. Kuo, X. L. Qi, S. K. Mo, R. G. Moore, D. H. Lu, M. Hashimoto, T. Sasagawa, S. C. Zhang, I. R. Fisher, Z. Hussain, and Z. X. Shen, Science 329, 659 (2010).

${ }^{4}$ C. Niu, Y. Dai, M. Guo, W. Wei, Y. Ma, and B. Huang, Appl. Phys. Lett. 98, 252502 (2011).

5 C.-Z. Chang, J. Zhang, X. Feng, J. Shen, Z. Zhang, M. Guo, K. Li, Y. Ou, P. Wei, L.-L. Wang, Z.-Q. Ji, Y. Feng, S. Ji, X. Chen, J. Jia, X. Dai, Z. Fang, S.-C. Zhang, K. He, Y. Wang, L. Lu, X.-C. Ma, and Q.-K. Xue, Science 340, 167 (2013).

6 R. Li, J. Wang, X.-L. Qi, and S.-C. Zhang, Nat. Phys. 6, 284 (2010).

7 W.-K. Tse and A. H. MacDonald, Phys. Rev. Lett. 105, 057401 (2010).

8 Xiao Zhang and Shou-Cheng Zhang, in Proceedings of the SPIE - The International Society for Optical Engineering, Vol. 8373 (2012) p. 837309, Micro- and Nanotechnology
Sensors, Systems, and Applications IV, 23-27 April 2012, Baltimore, MD, USA.

9 A. R. Mellnik, J. S. Lee, A. Richardella, J. L. Grab, P. J. Mintun, M. H. Fischer, A. Vaezi, A. Manchon, E. A. Kim, N. Samarth, and D. C. Ralph, Nature 511, 449 (2014).

10 Jing Wang, Biao Lian, and Shou-Cheng Zhang, Phys. Rev. Lett. 115, 036805 (2015).

11 Y. Xia, D. Qian, D. Hsieh, L. Wray, A. Pal, H. Lin, A. Bansil, D. Grauer, Y. S. Hor, R. J. Cava, and M. Z. Hasan, Nature Phys. 5, 398 (2009).

12 J.-M. Zhang, W. Zhu, Y. Zhang, D. Xiao, and Y. Yao, Phys. Rev. Lett. 109, 266405 (2012).

13 V. Kulbachinskii, A. Kaminskii, K. Kindo, Y. Narumi, K. Suga, P. Lostak, and P. Svanda, Physica B 311, 292 (2002).

14 J. Choi, S. Choi, J. Choi, Y. Park, H. Park, H. Lee, B. Woo, and S. Cho, Phys. Stat. Sol. (b) 241, 1541 (2004).

15 J. W. G. Bos, M. Lee, E. Morosan, H. W. Zandbergen, W. L. Lee, N. P. Ong, and R. J. Cava, Phys. Rev. B 74, 184429 (2006).

16 Y. S. Hor, P. Roushan, H. Beidenkopf, J. Seo, D. Qu, J. G. Checkelsky, L. A. Wray, D. Hsieh, Y. Xia, S.-Y. Xu, D. Qian, M. Z. Hasan, N. P. Ong, A. Yazdani, and R. J. 
Cava, Phys. Rev. B 81, 195203 (2010).

17 M. Watson, L. Collins-McIntyre, A. Coldea, D. Prabhakaran, L. R. Shelford, S. C. Speller, T. Mousavi, C. Grovenor, Z. Salman, S. R. Giblin, G. van der Laan, and T. Hesjedal, New J. Phys. 15, 103016 (2013).

${ }^{18}$ L. J. Collins-McIntyre, M. D. Watson, A. A. Baker, S. L. Zhang, A. I. Coldea, S. E. Harrison, A. Pushp, A. J. Kellock, S. S. P. Parkin, G. van der Laan, and T. Hesjedal, AIP Advances 4, 127136 (2014).

19 R. Yu, W. Zhang, H.-J. Zhang, S.-C. Zhang, X. Dai, and Z. Fang, Science 329, 61 (2010).

${ }^{20}$ P. P. J. Haazen, J. B. Laloe, T. J. Nummy, H. J. M. Swagten, P. Jarillo-Herrero, D. Heiman, and J. S. Moodera, Appl. Phys. Lett. 100, 082404 (2012).

${ }^{21}$ L. J. Collins-McIntyre, S. E. Harrison, P. Schoenherr, N.-J. Steinke, C. J. Kinane, T. R. Charlton, D. Alba-Veneroa, A. Pushp, A. J. Kellock, S. S. P. Parkin, J. S. Harris, S. Langridge, G. van der Laan, and T. Hesjedal, EPL 107, 57009 (2014).

22 A. I. Figueroa, G. van der Laan, L. J. Collins-McIntyre, S.-L. Zhang, A. A. Baker, S. E. Harrison, P. Schoenherr, G. Cibin, and T. Hesjedal, Phys. Rev. B 90, 134402 (2014).

23 A. I. Figueroa, G. van der Laan, L. J. Collins-McIntyre, G. Cibin, A. J. Dent, and T. Hesjedal, J. Phys. Chem. C 119, 17344 (2015).

${ }^{24}$ S. Li, S. Harrison, Y. Huo, A. Pushp, H. Yuan, B. Zhou, A. Kellock, S. Parkin, Y.-L. Chen, T. Hesjedal, and J. Harris, Appl. Phys. Lett. 102, 242412 (2013).

25 S. E. Harrison, L. J. Collins-McIntyre, S. Li, A. A. Baker, L. R. Shelford, Y. Huo, A. Pushp, S. S. P. Parkin, J. S. Harris, E. Arenholz, G. van der Laan, and T. Hesjedal, J. Appl. Phys. 115, 023904 (2014).

${ }^{26}$ S. E. Harrison, L. J. Collins-McIntyre, S.-L. Zhang, A. A. Baker, A. I. Figueroa, A. J. Kellock, A. Pushp, S. S. P.
Parkin, J. S. Harris, G. van der Laan, and T. Hesjedal, J. Phys.: Condens. Matter 27, 245602 (2015).

27 S. E. Harrison, L. J. Collins-McIntyre, P. Schönherr, A. Vailionis, V. Srot, P. A. van Aken, A. J. Kellock, A. Pushp, S. S. P. Parkin, J. S. Harris, B. Zhou, Y. L. Chen, and T. Hesjedal, Sci. Rep. XX, XXXX (2015).

28 S. Harrison, S. Li, Y. Huo, B. Zhou, Y.-L. Chen, and J. Harris, Appl. Phys. Lett. 102, 171906 (2013).

29 K. Virwani, S. E. Harrison, A. Pushp, T. Topuria, E. Delenia, P. Rice, A. Kellock, L. Collins-McIntyre, J. Harris, T. Hesjedal, and S. S. P. Parkin, Appl. Phys. Lett. 105, 241605 (2014).

30 See supplemental material at [URL will be inserted by AIP] for reciprocal space maps and band structure plots obtained at different photon energies.

31 A. Majid, A. Dar, and J. J. Zhu, J. Magn. Magn. Mater. 374, 676 (2015).

32 O. Zhong-Wen and R. Guang-Hui, Chin. Phys. B 22, 097501 (2013).

33 G. van der Laan, J. Phys.: Conf. Ser. 430, 012127 (2013).

${ }^{34}$ L. R. Shelford, T. Hesjedal, L. J. Collins-McIntyre, S. S. Dhesi, F. Maccherozzi, and G. van der Laan, Phys. Rev. B 86, 081304 (2012).

35 G. van der Laan and A. I. Figueroa, Coord. Chem. Rev. 277-278, 95 (2014).

36 B. T. Thole, G. van der Laan, J. C. Fuggle, G. A. Sawatzky, R. C. Karnatak, and J. M. Esteva, Phys. Rev. B 32, 5107 (1985).

37 G. van der Laan, Lect. Notes Phys. 697, 143 (2006).

38 S. E. Harrison, B. Zhou, Y. Huo, A. Pushp, A. J. Kellock, S. S. P. Parkin, J. S. Harris, Y. Chen, and T. Hesjedal, Appl. Phys. Lett. 105, 121608 (2014).

39 Y.-L. Chen, J. G. Analytis, J. H. Chu, Z. K. Liu, S.-K. Mo, X. L. Qi, H. J. Zhang, D. H. Lu, X. Dai, Z. Fang, S. C. Zhang, I. R. Fisher, Z. Hussain, and Z.-X. Shen, Science 325, 178 (2009). 